\title{
Stress Impairs Intentional Memory Control through Altered Theta Oscillations in Lateral Parietal Cortex
}

\author{
${ }^{\circledR}$ C.W.E.M. Quaedflieg, ${ }^{1,2}{ }^{\circledR}$ T.R. Schneider, ${ }^{3}{ }^{\oplus}$ J. Daume, ${ }^{3}$ A.K. Engel, ${ }^{3}$ and ${ }^{\circledR}$ L. Schwabe ${ }^{1}$ \\ ${ }^{1}$ Department of Cognitive Psychology, Institute of Psychology, Universität Hamburg, Hamburg 20146, Germany, ${ }^{2}$ Department of Neuropsychology and \\ Psychopharmacology, Maastricht University, Maastricht 6229 ER, The Netherlands, and ${ }^{3}$ Department of Neurophysiology and Pathophysiology, \\ University Medical Center Hamburg-Eppendorf, Hamburg 20246, Germany
}

Accumulating evidence suggests that forgetting is not necessarily a passive process but that we can, to some extent, actively control what we remember and what we forget. Although this intentional control of memory has potentially far-reaching implications, the factors that influence our capacity to intentionally control our memory are largely unknown. Here, we tested whether acute stress may disrupt the intentional control of memory and, if so, through which neural mechanism. We exposed healthy men and women to a stress $(n=27)$ or control $(n=26)$ procedure before they aimed repeatedly to retrieve some previously learned cue-target pairs and to actively suppress others. While control participants showed reduced memory for suppressed compared with baseline pairs in a subsequent memory test, this suppression-induced forgetting was completely abolished after stress. Using magnetoencephalography (MEG), we show that the reduced ability to suppress memories after stress is associated with altered theta activity in the inferior temporal cortex when the control process (retrieval or suppression) is triggered and in the lateral parietal cortex when control is exerted, with the latter being directly correlated with the stress hormone cortisol. Moreover, the suppression-induced forgetting was linked to altered connectivity between the hippocampus and right dorsolateral prefrontal cortex (PFC), which in turn was negatively correlated to stress-induced cortisol increases. These findings provide novel insights into conditions under which our capacity to actively control our memory breaks down and may have considerable implications for stress-related psychopathologies, such as posttraumatic stress disorder (PTSD), that are characterized by unwanted memories of distressing events.

Key words: cortisol; memory; memory suppression; stress; theta

Significance Statement

It is typically assumed that forgetting is a passive process that can hardly be controlled. There is, however, evidence that we may actively control, to some extent, what we remember and what we forget. This intentional memory control has considerable implications for mental disorders in which patients suffer from unwanted (e.g., traumatic) memories. Here, we demonstrate that the capacity to intentionally control our memory breaks down after stress. Using magnetoencephalography (MEG), we show that this stress-induced memory control deficit is linked to altered activity in the lateral parietal cortex and the connectivity between the hippocampus and right prefrontal cortex (PFC). These findings provide novel insights into conditions under which memory control fails and are highly relevant in the context of stress-related psychopathologies.

Received Dec. 6, 2019; revised July 9, 2020; accepted July 13, 2020.

Author contributions: C.W.E.M.O. and L.S. designed research; C.W.E.M.O. and T.R.S. performed research; C.W.E.M.Q. J.D., and T.R.S. analyzed data; C.W.E.M.Q and L.S. wrote the paper, T.R.S., J.D., and A.K.E. provided critical revisions.

This work was supported by The Netherlands Organization for Scientific Research (Nederlandse Organisatie voor Wetenschappelijk Onderzoek, NW0) Grants 446-15-003 and VI.Veni.191G.004 (to C.W.E.M.Q.) and by the German Research Foundation (Deutsche Forschungsgemeinschaft, DFG) Grant SFB 936, projects B10 (to L.S.) and A3 (T.R.S. and A.K.E.). We are especially thankful to Hanna Stoffregen for her help in collecting the data. The authors declare no competing financial interests.

Correspondence should be addressed to C.W.E.M. Quaedflieg at conny.quaedflieg@maastrichtuniversity.nl or L. Schwabe at lars.schwabe@uni-hamburg.de.

https://doi.org/10.1523/JNEUROSCI.2906-19.2020

Copyright $\odot 2020$ the authors

\section{Introduction}

While we want to keep some memories for the rest of our lives, others are so painful that we wish to forget them forever. For long, forgetting was assumed to be a passive process that cannot be intentionally controlled (Ebbinghaus, 1885; Schacter, 1999). More recent research, however, suggests that we may exert control over our episodic memory through control processes (Anderson and Hanslmayr, 2014). Intentional memory control is assumed to be highly adaptive, helping us to remember valued memories while suppressing memories that pose a threat to our integrity or well-being (Engen and Anderson, 2018; Norby, 2018). The failure to intentionally control memories, however, has been linked to disorders' such as posttraumatic stress 
disorder (PTSD; Catarino et al., 2015), depression (Joormann et al., 2009), and anxiety (Gómez-Ariza et al., 2013).

Intentional memory control has been investigated using the think/no-think paradigm in which participants aim repeatedly to actively suppress some learned associations and to retrieve others (Anderson and Green, 2001; Benoit et al., 2016). The comparison of suppressed and retrieved items showed that intentional memory suppression relies on an executive control network guided by the right lateral prefrontal cortex (PFC), in interaction with parietal and medial temporal areas (Benoit and Anderson, 2012; Paz-Alonso et al., 2013; Depue et al., 2016). Moreover, stronger functional coupling between these regions has been linked to successful forgetting (Paz-Alonso et al., 2013; Benoit et al., 2015). Using electroencephalography, it has been demonstrated that intentional memory suppression is in part achieved by a twostage inhibitory control mechanism that is evident in the theta frequency (4-7 Hz; Ketz et al., 2014). Specifically, control processes before suppression trials were characterized by event-related theta synchronization in the PFC while successful suppression of target memories was characterized by theta desynchronization in the medial temporal lobe (Waldhauser et al., 2015). In addition, successful memory suppression is characterized by frontal and parietal theta synchronization thought to reflect the monitoring of retrieval attempts (Depue et al., 2013).

Acute stress is known to have a major impact on memory. Stress may modulate memory formation and retrieval in a time-dependent manner, closely linked to the temporal profile of action of major stress mediators in the brain, in particular noradrenaline and glucocorticoids (Joëls et al., 2011; Schwabe et al., 2012; Quaedflieg and Schwabe, 2018). Stress is thought to exert these effects by altering the functioning of those frontal and temporal brain areas that are implicated in intentional memory control (Pruessner et al., 2008; Qin et al., 2009; Hermans et al., 2014; Quaedflieg et al., 2015; Vogel et al., 2018). Furthermore, there is evidence for reduced theta oscillations, a key characteristic of intentional memory control, during cognitive tasks under stress (Gärtner et al., 2014, 2015). Although stress-induced deficits in intentional memory control would be highly relevant in the context of stressrelated psychopathologies characterized by dysfunctional memories, this hypothesis has not been tested so far.

Here, we aimed to test whether acute stress may interfere with the capacity to intentionally control episodic memories. We predicted that stress would impair the intentional control of memory via altered theta synchronization in frontal, parietal and medial temporal areas. To test this hypothesis, we presented participants first a series of face-word associations and then exposed them to a stress or control manipulation. Shortly thereafter, participants engaged in a think/ no-think procedure in which they repeatedly tried to actively retrieve or suppress the previously learned associations while magnetoencephalography (MEG) was recorded. The impact of this intentional control attempt on the actual memory performance was tested later on. As theta oscillations have been implicated in memory retrieval (Klimesch, 1999), maintenance (Nyhus and Curran, 2010), and intentional memory control (Ketz et al., 2014; Waldhauser et al., 2015), and a stress-induced reduction in theta oscillations has been demonstrated (Gärtner et al., 2014, 2015), we focused on MEG activity in the theta band as neural marker of stress-induced changes in intentional memory control.

\section{Materials and Methods}

\section{Participants and experimental design}

Exclusion criteria were screened in a standardized interview before participation and comprise a body mass index (BMI) below 18 or above $27.5 \mathrm{~kg} / \mathrm{m}^{2}$, any current or lifetime psychiatric or neurologic disorders, current or history of drug abuse, nicotine consumption, medication intake or vaccination within the past month. Further, women being pregnant or taking hormonal contraceptives were excluded from participation and women were not tested during their menses. These criteria were applied as it is known that these factors influence the cortisol response (Strahler et al., 2017). The a priori power calculation with $\mathrm{G}^{*}$ Power $(\alpha=0.05,1-\beta=0.90$; Faul et al., 2007) indicated a total sample size for the decisive condition $\times$ stimulus type interaction effect of $N=44$. We tested in total 67 healthy young adults ( 32 men, 35 women; age range $18-35$, mean age $=25.06$ years, $S D=3.72$ years). One participant was excluded because of depressive symptoms (score above the clinical cutoff on the Becks Depression Inventory; Beck et al., 1996), and four participants were excluded because they were classified as behavioral outliers based on a $z$ score smaller than -2.5 on recall in the final memory test. Because of technical difficulties behavioral data were missing for two participants and MEG data were missing for seven further participants, thus leaving a sample of 53 participants for the final analysis ( $n=26$ in the control group, $n=27$ in the stress group). Participants were asked to refrain from physical exercise, beverages other than still water, and meals in the $2 \mathrm{~h}$ before testing. Participants were pseudo-randomly assigned to one of the two experimental groups (stress or control). If participants had participated in the stress condition of the Trier Social Stress Test (TSST) before, they were allocated to the control condition. All testing took place in the afternoon between 12:30 and 6 P.M. to control for the circadian rhythm of cortisol. All participants provided written informed consent and received a moderate monetary compensation for participation. The study procedure was approved by the standing ethics committee of the Faculty of Psychology and Human Movement, Hamburg University.

In a 2 (condition: stress vs no-stress) $\times 3$ (stimulus: think, no-think, baseline) mixed design, participants were semi-randomly assigned to a stress or control condition.

\section{Stimulus materials and experimental paradigm}

In order to assess the intentional control of memory, we used in the think/no-think paradigm (Hanslmayr et al., 2009; Fig. 1). Stimuli were 33 cue-target pairs with neutral faces as cues and neutral German nouns as targets. All face pictures displayed white faces and were drawn from the AR Face Database (Martinez and Benavente, 1996). The words were semantically unrelated neutral five-letter words selected on word frequency from a semantically categorized German word database (Schwibbe, 1988). Three face-word pairs were used as practice items for the think/no-think phase and were excluded from the analysis. From the remaining face-word pairs, 10 were used as baseline items and not presented during the think/no-think phase, 10 were used as think items, and 10 were used as no-think items in the think/no-think phase.

\section{Learning phase}

Participants were instructed to memorize 33 face-word pairs. The 33 face-word pairs were each presented once per run for $4 \mathrm{~s}$ on a computer screen with four runs in total. Each run was followed by a cued recall test, during which the face of a face-word pair was presented for $4 \mathrm{~s}$ as a memory cue for the target word. Participants were instructed to use the face as a memory cue to recall the associated word, and to speak out the word as soon as they remembered the word. The experimenter scored the words as correct or incorrect.

\section{Think/no-think phase}

Participants performed the think/no-think manipulation with 10 think and 10 no-think face-word pairs chosen randomly from the 33 faceword pairs. For think trials, participants were instructed to think of the target word when the memory cue (face) was presented. In no-think trials, participants were instructed to avoid thinking about the previously learned item by pushing the target item out of consciousness. 


\section{THINK / NO-THINK PARADIGM}

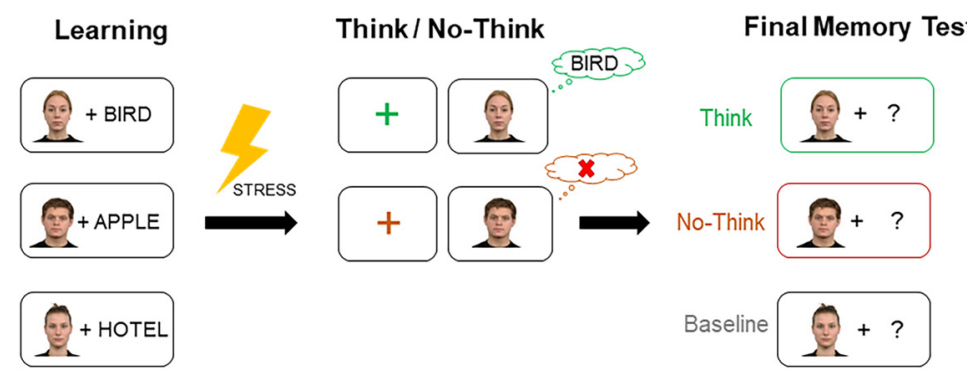

Figure 1. Think/no-think paradigm. Three phases of the think/no-think paradigm. In the learning phase, participants learned 33 face-word pairs. Three face-word pairs were used to practice the think/no-think manipulation. In the think/no-think phase, a green or red cross indicated whether participants were asked to actively recall (think items) or actively suppress the word stimulus (no-think items), when presented with the corresponding face stimulus. The recall or suppression was repeated 14 times for the respective items. In the final memory test, memory for all 30 face-words pairs was tested. Stimuli in the baseline condition were not presented during the think/no-think phase.

Participants were told to focus on the memory cue, and they were explicitly asked not to generate other associations to the memory cue or distractive thoughts or memories. Each trial was initiated by a white fixation cross of variable duration (1.0-1.5 s), which turned either green or red for $1 \mathrm{~s}$ to cue for a think (green) or a no-think (red) item, respectively (i.e., initiation phase). This was immediately followed by a 4 -s display of the face memory cue and participants were asked to suppress or retrieve the target word (i.e., intentional control phase). During the think/no-think phase, each of the 10 think and 10 no-think face-word pairs was presented 14 times, always with the same instruction to either actively retrieve or suppress the referring association, resulting in a total of 280 trials. The face-word pairs were presented pseudo-randomly with a maximum of three consecutive trials of the same trial type. To familiarize participants with the no think procedure, participants performed 15 no-think practice trials for three of the previously encoded face-word pairs (not included in further analysis).

\section{Final memory test}

The effects of the think/no-think manipulation was assessed in the final memory test. Participants performed a cued-recall test including all 30 previously studied targets from phase 1 . The face of a face-word pair was presented for $4 \mathrm{~s}$ as a memory cue for the target word. Participants were instructed to use the face as a memory cue to recall the associated word, and to speak out the word as soon as they remembered the word. The experimenter scored the words as correct or incorrect.

\section{Stress and control manipulation}

Participants in the stress condition were exposed to the TSST (Kirschbaum et al., 1993), a gold standard in experimental stress research, after the learning phase and before the think/no-think phase. In brief, the TSST mimics a job interview tailored to the participants' interests that consisted of a 3-min preparation period, a 5-min free speech, and a 5-min mental arithmetic task (counting backwards from 2043 in steps of 17). All tasks were performed in front of a panel, dressed in white lab coats, and composed of a male and female experimenter that were introduced as experts in behavioral analysis. The panel was rather cold and non-reinforcing and took notes. Furthermore, participants were videotaped during the TSST and could see themselves on a screen placed behind the panel.

Participants in the control condition underwent a standardized control procedure in which they were asked to talk for $5 \mathrm{~min}$ about a recent movie they had seen or a book they had read, followed by a simple counting task, all without a panel and without being videotaped.

In order to assess the effectiveness of the stress manipulation, we took subjective ratings, blood pressure measurements, and saliva samples at several time points across the experiment. Subjective stress was measured using three $100-\mathrm{mm}$ visual analogue scales (VAS) on which participants rated how stressful, unpleasant, and difficult they had perceived the TSST (anchors: $0=$ "not at all"; 100 = "extremely") immediately after the TSST or control procedure. Furthermore, we measured mood changes with the negative affect subscale of the state positive and negative affect schedule (PANAS; Watson et al., 1988). Negative mood was assessed at baseline and after the TSST. For three participants the PANAS scores were missing. Blood pressure (arm cuff: Omron Healthcare Europe BV) was measured shortly before $\left(\mathrm{T}_{-1}\right)$, during $\left(\mathrm{T}_{10}\right)$, and immediately after the TSST $\left(\mathrm{T}_{15}\right)$. Saliva samples were obtained shortly before and immediately after the TSST $\left(\mathrm{T}_{15}\right), 15 \mathrm{~min}$ after the TSST $\left(\mathrm{T}_{30}\right)$, as well as before the beginning $\left(\mathrm{T}_{70}\right)$ and after the end of the think/no-think phase $\left(\mathrm{T}_{105}\right)$. At the end of data collection, we analyzed from saliva the free fraction of cortisol using a luminescence assay (IBL).

\section{MEG acquisition and analysis}

MEG data were obtained during the think/no-think phase using a 275-channel whole-head system (Omega 2000, CTF Systems Inc.) with a sampling rate of $1200 \mathrm{~Hz}$. Additional $\mathrm{Ag} / \mathrm{AgCl}$-electrodes were applied to measure horizontal and vertical electrooculogram (EOG) and electrocardiogram (ECG). The head position relative to MEG sensors was monitored on-line during the whole recording and corrected between experimental blocks as soon as the movement exceeded $5 \mathrm{~mm}$ using three fiducial points (nasion, left and right external ear canal).

Preprocessing and analysis of the MEG data were done in MATLAB (version R2011b; The MathWorks) using the FieldTrip toolbox (Oostenveld et al., 2011). Data were down sampled to $400 \mathrm{~Hz}$, filtered between 0.5 and $170 \mathrm{~Hz}$ (Butterworth filter, low-pass filter order 4, highpass filter order 3), and for line-noise with band-stop filters for 50, 100, and $150 \mathrm{~Hz}$ (Butterworth filter, low-pass filter order 4, high-pass filter order 3), epoched into 4.5-s time bins ( $-1-3.5 \mathrm{~s}$ with respect to onset of the think/no-think precue). A two-step procedure was performed to remove artifacts using independent component analysis (ICA), in accordance with previous studies (Debener et al., 2005). First, epochs containing non-stereotyped artifacts (e.g., SQUID jumps, bypassing cars, muscle artefacts) were semi-automatically detected and rejected from further analysis. Then, extended infomax ICA (stop criterion: weight change $<10^{-7}$ ) was applied. Independent components corresponding to eye blinks and heart beat were identified via topographic plots and via correlation with EOG and ECG signals. Components correlating $>0.9$ with either EOG or ECG were rejected. Components correlating 0.6-0.9 were rejected only after a second visual inspection of the topographic plot. Application of this procedure ensured that $74.8 \%$ of all recorded trials (think mean: $75.5 \%$, range: $32-97 \%$; no-think mean: $74 \%$, range: $32-$ 97\%) could be retained.

Spectral decomposition was performed using sliding Hanning windows $(2-30 \mathrm{~Hz}, 1-\mathrm{Hz}$ steps, five-cycle window). The single trials were log-transformed (Grandchamp and Delorme, 2011; Smulders et al., 2018 ) and baseline corrected ( $-1000-0 \mathrm{~ms}$ precue). Theta was defined as $4-7 \mathrm{~Hz}$ and was then averaged per stimulus type (think and no-think) across participants in the stress and control condition, respectively.

In accordance with previous studies (Schneider et al., 2018), cortical sources were estimated using dynamic imaging of coherent sources (DICS; Gross et al., 2001), a linear beamforming technique implemented in Fieldtrip. In order to construct head models for source localization, we used individual T1-weighted structural magnetic resonance imaging (MRI; Siemens Magnetom Prisma) data with $1-\mathrm{mm}^{3}$ voxel size for 51 participants, while the standard MNI 152 brain template was used for two participants for whom MRI data were not available. Individual MEG sensor positions were aligned to the MR images based on three fiducials (left and right acoustic meatus, nasion) using rigid body transformation. Individual MRI data were segmented using the SPM12b software. Head models were derived from individual MR images using the single-shell volume conductor model (Nolte, 2003). A source model was defined using a regular grid, with regular $8 \mathrm{~mm}$ spacing, in the $152 \mathrm{MNI}$ template brain space. This source model was warped into the individual 
headspace using SPM functions implemented in Fieldtrip, bases on the individuals' MRI when available. Leadfield matrices were calculated for each participant using the individual MEG sensor positions aligned to the individual head model and the individual source grid (when available). This procedure allows later to average source activity across participants in the MNI template brain space.

Cross-spectral density matrices of the MEG data were computed for the initiation window lasting from 0 till $1000 \mathrm{~ms}$ and the intentional control window lasting from 1000 to $3000 \mathrm{~ms}$. Common filters were computed by averaging the cross-spectral density matrices across all time windows, both stimulus types and conditions. To obtain power estimates in each voxel, the common filters were multiplied with the cross-spectral density matrix of each stimulus type (think and no-think) and time window separately. In order to minimize the number of statistical tests, source power values were calculated for two regions of interest (ROIs), right dorsolateral PFC (dlPFC; BA 46) and right lateral inferior parietal lobe (IPL; BA 39c), which have been implicated in executive control in general and memory control in particular in previous studies (PazAlonso et al., 2013; Anderson and Hanslmayr, 2014; Depue et al., 2016), using the Brainnetome atlas (Fan et al., 2016).

Functional connectivity was estimated between the atlas-based right hippocampus (AAL; Tzourio-Mazoyer et al., 2002) and the two ROIs implicated in executive control using the imaginary part of coherency (Nolte et al., 2004). Imaginary coherence was computed for all possible combinations of source points and subsequently averaged within the different ROIs. Imaginary coherence measures the synchronization between two ROIs which are phase-lagged at the specific frequency and thus robust to effects of volume conduction. The mean values between the ROIs for each subject for the think versus no-think contrast were used for the statistical comparison of conditions. For source localization and quantification of imaginary coherence, trial numbers for the learned think and no-think word-face pairs were stratified to obtain comparable signal-to-noise ratios between conditions.

\section{Statistical analysis}

Data analysis were performed using SPSS Statistics for Windows, version 25 (SPSS Inc.). The data were checked for normality and outliers. $P$ values were Greenhouse-Geisser corrected when required. All reported $p$ values are two-tailed, unless stated otherwise.

Effectiveness of the stress induction procedure on salivary cortisol levels were assessed using repeated measures ANOVA with time (four levels) as the within-subject variable and condition (two levels: stress and control) as the between-subjects variable. Cortisol data were logtransformed because of a typical skewness of the data. Changes in subjective stress and negative mood were evaluated using independent samples $t$ tests with condition (stress, control) as between-subjects variable.

Face-word pairs need to be learned to be able to intentionally control the memory of it. Thus, in line with previous studies (Anderson et al., 2004; Hanslmayr et al., 2009), data analysis was based only on those face-word pairs that were correctly recalled at least once during the training phase. First, we ran a repeated measures ANOVA including all three stimulus types. Based on our a priori hypothesis of a stress-induced impairment in memory control, one-tailed non-parametric MannWhitney $U$ follow-up tests were performed comparing performance between conditions for the suppression-induced forgetting (baselineno-think) and retrieval (baseline-think) index. Second, we compared performance for think and no-think trials, (i.e., total control effect) with one-tailed non-parametric Mann-Whitney $U$ tests and follow-up onetailed Wilcoxon signed-rank test per condition.

For the MEG data, we first assessed an average of spectral power in the theta frequency for the initiation (0-0.9 s) and intentional control (1.0-3.0 s) phase. We tested condition differences between think and no-think whole-brain at the sensor level using cluster-based permutation tests $(10,000$ permutations using "maxsum" option in ft_statistics_montecarlo to correct for multiple comparisons; Maris and Oostenveld, 2007). The channel-frequency-time samples were clustered at an $\alpha$ level of 0.01. Clusters with a Monte Carlo $p$ value of 0.05 and less are reported significant. Significant condition differences at the sensor level were followed-up per item type and by source localization. Next, we assessed
Table 1. Subjective and physiological measurements of stress induction through the TSST

\begin{tabular}{llll}
\hline Subjective stress (VAS) & Stressful & Unpleasant & Difficult \\
\hline Control & $20.69(4.30)$ & $16.88(4.57)$ & $14.85(3.27)$ \\
Stress & $58.26(4.22)$ & $56.14(4.49)$ & $57.22(3.22)$ \\
Negative mood (PANAS) & Baseline & After & \\
Control & $10.54(0.34)$ & $10.63(0.56)$ & \\
Stress & $11.77(0.33)$ & $15.08(0.54)$ & \\
Diastolic blood pressure & Baseline & During & After \\
Control & $80.62(1.49)$ & $82.71(1.77)$ & $82.92(1.77)$ \\
Stress & $83.22(1.42)$ & $93.22(1.73)$ & $90.78(1.59)$ \\
\hline
\end{tabular}

Subjective stress was measured using three 100 -mm visual VAS on which participants rated how stressful, unpleasant, and difficult they had perceived the TSST (anchors: $0=$ "not at all"; $100=$ "extremely") immediately after the TSST or control procedure. Blood pressure measurements were taken shortly before at baseline $(t=-1)$, during $(t=0)$, and immediately after $(t=15)$ the TSST.

theta source activity in the two ROIs using independent samples $t$ tests with condition (stress vs control) as between subject variable. Finally, the effect of stress on functional connectivity between the hippocampus and the two ROIs was assessed per stimulus type using the imaginary part of coherency. The values were subjected to an independent samples $t$ test with condition (stress vs control) as between subject variable. To examine whether the impaired intentional memory control could be directly linked to the stress response, theta power, and theta connectivity, Pearson's correlations were computed.

All data and code is available at https://osf.io/36g5f/?view_only= eecd3cd5060d4146b1ff5a3275f9c94d.

\section{Results}

\section{Successful stress induction}

Directly after the learning phase, stress was induced in half of the participants using the TSST whereas the other half of the participants underwent a non-stressful control manipulation. Significant increases in subjective stress ratings, blood pressure, and salivary cortisol confirmed the successful stress induction through the TSST. Participants in the stress condition experienced the experimental manipulation as significantly more stressful, unpleasant, and difficult than participants in the control condition (all $p s<0.001$; all $\eta_{\mathrm{p}}^{2}>0.42$; Table 1). Negative mood, as measured with the negative affect subscale of the state PANAS, increased from baseline to posttreatment in the stress condition but not in the control condition $\left(F_{(1,48)}=22,45\right.$, $p<0.001, \eta \mathrm{pi}_{\mathrm{p}}^{2}=0.32$; Table 1$)$. Furthermore, systolic and diastolic blood pressure were elevated in response to the TSST but not in response to the control manipulation (time $\times$ condition interaction systolic blood pressure: $F_{(2,102)}=20.90, p<0.001$, $\eta_{\mathrm{p}}^{2}=0.29$; see Fig. $2 A$, diastolic blood pressure: $F_{(2,102)}=14.38$, $p<0.001, \eta_{\mathrm{p}}^{2}=0.22$; Table 1). Follow-up tests showed that whereas groups did not differ at baseline (both $p s>0.21$ ), participants exposed to the TSST had significantly higher blood pressure than participants in the control group during $\left(\mathrm{T}_{10}\right.$ systolic: $t_{(51)}=5.14, p<0.001, d=1.32$; diastolic: $t_{(51)}=4.25, p<0.001$, $d=1.12)$ and directly after the TSST $\left(\mathrm{T}_{15}\right.$ systolic: $t_{(51)}=3.49$, $p=0.001, d=0.80$; diastolic: $\left.t_{(51)}=3.46, p=0.001, d=0.74\right)$. Finally, salivary cortisol increased after the exposure to the TSST but not after the control manipulation (time $\times$ group interaction: $\left.F_{(4,204)}=15.78, p<0.001, \eta_{\mathrm{p}}^{2}=0.24\right)$. As displayed in Figure $2 B$, groups did not differ at baseline $(p=0.716)$, but the stress group had significantly higher cortisol concentrations immediately $\left(t_{(51)}=3.67, p=0.001, d=0.90\right)$ and $15 \mathrm{~min}$ after the experimental treatment $\left(t_{(51)}=4.95, p<0.001, d=1.24\right)$ as well as before the think/no-think phase $\left(t_{(51)}=2.47, p=0.017\right.$, $d=0.66$ ). Ninety minutes after the stress induction, before the 
A

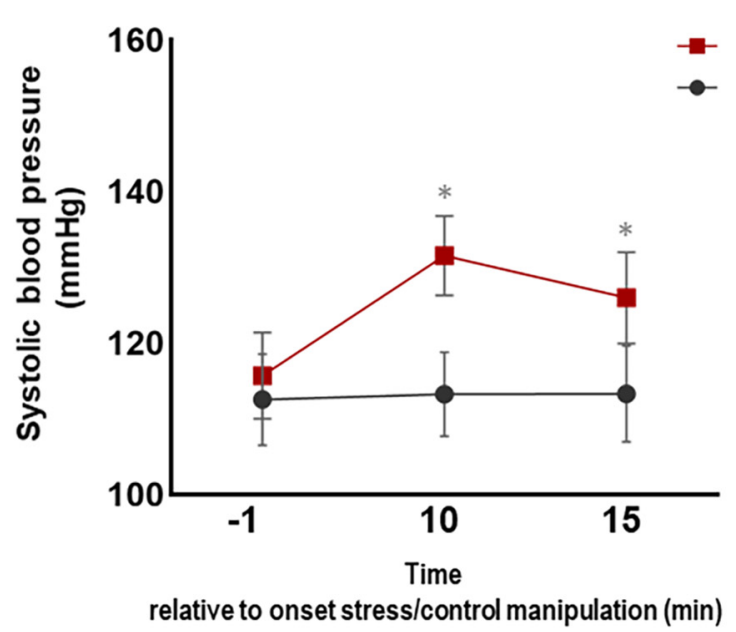

B

\section{Cortisol response}

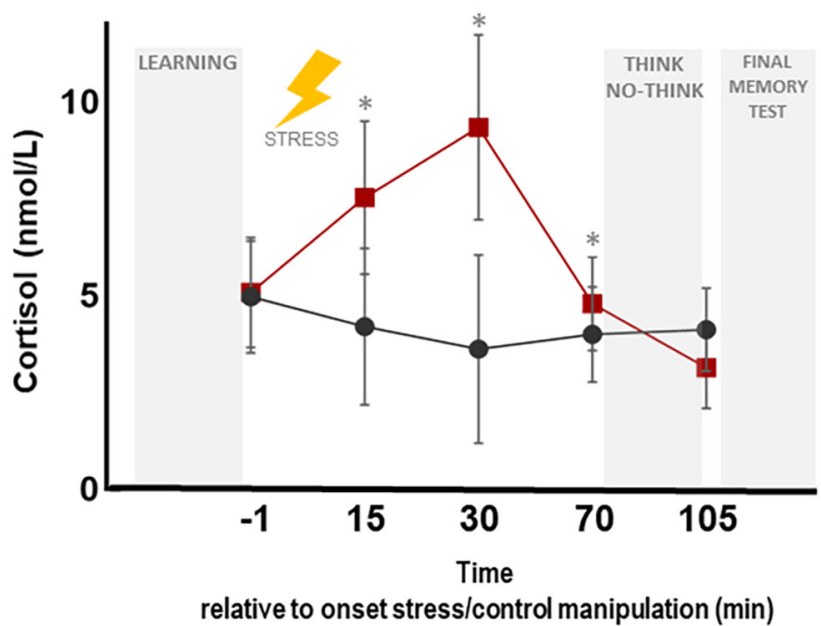

Figure 2. Effectiveness of the stress manipulation through the TSST. $A$, Significant increases in blood pressure during and after the TSST confirmed the successful stress induction. Blood pressure measurements were taken shortly before $(t=-1)$, during $(t=10)$, and immediately after $(t=15)$ the TSST. $\boldsymbol{B}$, The stress group had significantly higher cortisol concentrations immediately and $15 \mathrm{~min}$ after the experimental or control treatment as well as before the think/no-think phase. Cortisol samples were obtained shortly before $(t=-1)$ and immediately after the TSST $(t=15), 15 \mathrm{~min}$ after the TSST $(t=30)$, as well as before the beginning $(t=70)$ and after the end $(t=105)$ of the think/no-think phase. Data show means $( \pm S E) ;{ }^{*} p<0.02$.

final memory test, groups did not differ in their cortisol concentrations $\left(t_{(51)}=-0.18, p=0.856, d=-0.05\right)$.

\section{Stress impairs the intentional control of memory Initial memory encoding}

To assess intentional memory control, we used the think/nothink paradigm, which consists of a learning phase, a think/nothink phase, and a final memory test. In the learning phase, participants learned 33 face-word pairs. To ensure that the stress $(n=27)$ and control $(n=26)$ groups learned the face-word pairs to a comparable extent, recall performance was analyzed with repeated measures ANOVA. Participants in both conditions learned the face-word pairs very well $\left(F_{(2,204)}=487.48, p<0.001\right.$, $\left.\eta_{\mathrm{p}}^{2}=0.91\right)$, without any difference between conditions or stimulus types (time $\times$ condition interaction as well as main effects of condition or stimulus type: all $p s>0.167$; Fig. $3 A$ ).

\section{Intentional memory control}

The performance in the control condition in the final memory test was overall very high (baseline: $-x=90.04$, SE $=2.28$, nothink: $-x=86.13, \mathrm{SE}=2.67$; think: $-x=94.08, \mathrm{SE}=2.52$ ), in line with previous studies using the think/no-think paradigm (Anderson and Green, 2001; Hanslmayr et al., 2009; Benoit and Anderson, 2012; Depue et al., 2013; Paz-Alonso et al., 2013; Ketz et al., 2014; Benoit et al., 2015; Waldhauser et al., 2015). In a first step, we compared the memory for think, no-think and baseline face-word pairs between groups and this analysis revealed a significant interaction between stimulus type and condition $\left(F_{(1.817,92.669)}=4.51, p=0.016, \eta_{\mathrm{p}}^{2}=0.08\right.$; stress condition: baseline: $-x=91.33$, $\mathrm{SE}=2.24$, no-think: $-x=93.69$, $\mathrm{SE}=2.62$; think: $-x=89.41, \mathrm{SE}=2.47)$. Follow-up analyses revealed that stress interfered in particular with the active suppression of the face-word pairs (baseline-no-think: $U_{(53)}=252.500 ; p=0.0345$, one-tailed; Fig. $3 B$ ), whereas the active retrieval of memories, as reflected in the retrieval index (baseline-think), was not significantly affected by stress $\left(U_{(53)}=248.500 ; p=0.0725\right.$, one-tailed; Fig. $3 C$ ). In a next step, we analyzed the difference in performance for think and no-think items, as this total control effect is commonly used as index of intentional memory control
(Anderson and Levy, 2009; Anderson and Huddleston, 2012; Depue et al., 2016), and reflects the contrast used in the MEG analysis, which focused on the think/no-think phase during which no baseline items are presented. The analysis of the total control effect showed that stress impaired, in line with our directed a priori hypothesis, the ability to intentionally control memory $\left(U_{(53)}=223.00 ; p=0.010\right.$, one-tailed; Fig. $\left.3 D\right)$. More specifically, while participants in the control condition showed efficient intentional memory control reflected in a significant different median for think minus no-think face-word pairs from 0 $\left(Z_{(26)}=141.00 ; p=0.008\right.$, one-tailed; Fig. $\left.3 D\right)$, stress abolished this intentional memory control effect, i.e., stressed participants showed no difference in their memory for think and no-think face-word pairs from $0\left(Z_{(27)}=41.00 ; p=0.210\right.$, one-tailed, $d=0.21$; Fig. $3 D$ ).

\section{Stress reduces parietal theta activity during intentional memory control}

In order to unravel the neural mechanisms underlying the observed impairment of intentional memory control after stress, we recorded oscillatory brain activity using wholehead MEG while participants tried to actively retrieve or suppress the previously encoded associations (i.e., during the think/no-think phase). Each trial consisted of an initiation phase in which a red or green fixation cross signaled the required mode of memory control, and the actual intentional control phase in which the face memory cue was presented and participants were asked to suppress or retrieve the target memory (Fig. 1). Note that baseline items were not shown during the think/no-think phase 2 while brain activity was recorded.

First, we assessed whole-brain permutation corrected sensor spectral power for the initiation phase. To identify neural systems involved in intentional memory, we contrasted, in line with previous studies (Hanslmayr et al., 2009, 2012; Ludowig et al., 2010; Benoit and Anderson, 2012; Depue et al., 2013, 2016; PazAlonso et al., 2013; Ketz et al., 2014; Waldhauser et al., 2015, 2018; Oehrn et al., 2018), theta power during think and no-think trials. Cluster-based permutation tests revealed that stress 


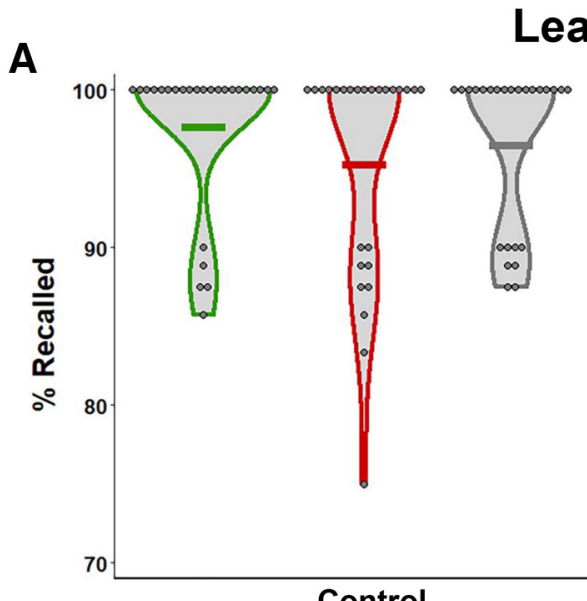

Control

Learning

Final memory test

B

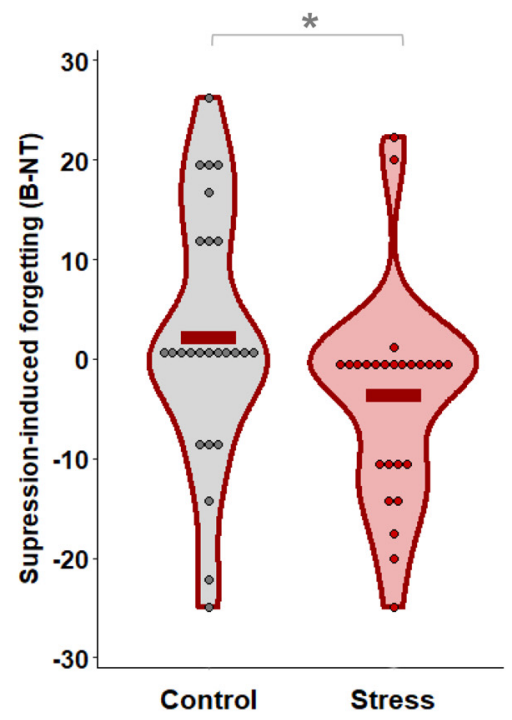

C

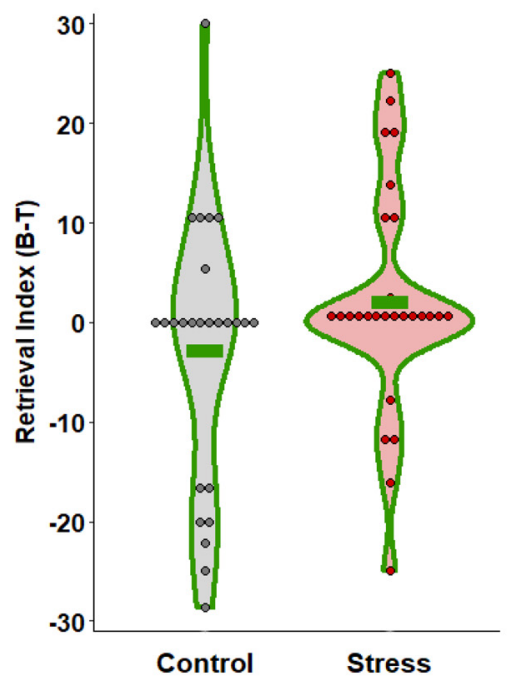

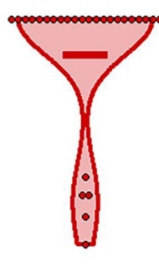

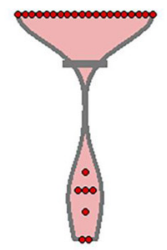

- THINK (T)

- NO-THINK (NT)

- BASELINE (B)

\section{Stress}

D

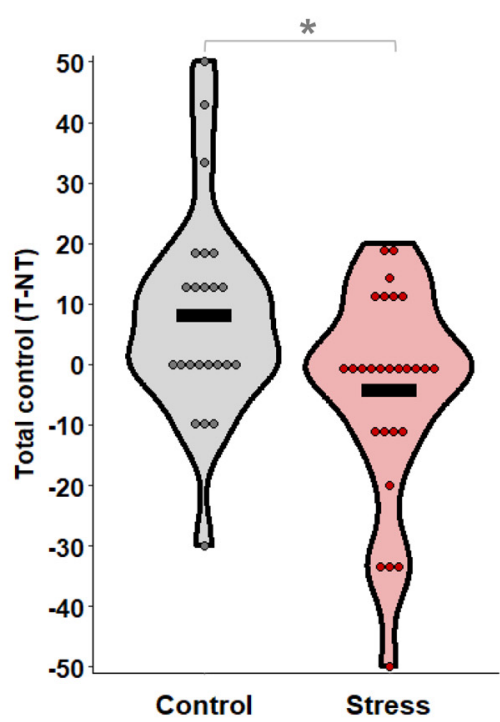

Figure 3. Think/no-think behavioral data. $\boldsymbol{A}$, Participants (control $n=26$; stress $n=27$ ) learned the face-word associations very well. $\boldsymbol{B}$, Stress-induced deficit in intentional memory control primarily owing to a disruptive effect of stress on intentional suppression of memories (baseline-no-think). $\boldsymbol{C}$, Stress did not significantly influence intentional retrieval (baseline-think). $\boldsymbol{D}$, Stress reduced the total control effect (i.e., think-no-think). Violin plots display the distribution of the data, group means (indicated by the bar), and individual data points; $\mathrm{B}=$ baseline word pair, $\mathrm{NT}=$ no-think word pair, $\mathrm{T}=$ think word pair; ${ }^{*} p<0.05$.

modulated theta power for think versus no-think items in the initiation phase (cluster $p=0.034$; Fig. $4 A, B$ ). Source localization revealed that this power difference originated in the inferior temporal lobe (Fig. 4B). Higher cortisol levels, suppression-induced forgetting or retrieval were not associated with theta power during the initiation phase (all $p s>0.101$ ).

For the intentional control phase, cluster-based permutation tests revealed a stress-induced modulation of theta power for the total control effect (think vs no-think: cluster $p=0.002$; Fig. $4 C, D)$. Source localization revealed that this power difference originated in the left lateral inferior parietal cortex. Moreover, bivariate Pearson correlations, revealed a condition difference between theta activity and the retrieval index (B-T; $Z=1.982$, $p=0.042$ ). In the control condition, higher theta activity for think versus no-think items was associated with increased recall of think items compared with baseline items (baseline-think; $\left.r_{(26)}=-0.408, p=0.043\right)$, corroborating the suggestion that the parietal cortex supports memory retrieval (Shimamura, 2011). This association was opposite and not significant in the stress condition $\left(r_{(27)}=0.144, p=0.473\right)$. Higher cortisol levels, were not associated with theta power during the intentional control phase (all $p s>0.095$ ).

Previous studies indicated that the right dlPFC and right IPL are the key areas that regulate activity in the medial temporal lobe during intentional memory control (Paz-Alonso et al., 2013; Anderson and Hanslmayr, 2014; Depue et al., 2016). Thus, to better understand the mechanisms underlying the effect of stress on intentional memory control, we next used a ROI approach and restricted the analysis at the source level to those two key structures. This analysis revealed a significant modulation of theta power in the right IPL during the intentional control phase $\left(t_{(51)}=2.70, p=0.010, d=0.756\right)$. Moreover, bivariate Pearson correlations revealed a condition difference between IPL theta activity and the cortisol increase (AUCi; $\mathrm{Z}=1.646, p=0.05$ ). In the stress condition, a higher cortisol increase was associated with decreased right IPL theta activity for think versus no-think items $\left(r_{(27)}=-0.427, p=0.027\right)$, while this association was absent in the control condition $\left(r_{(26)}=0.024, p=0.909\right.$; Fig. $\left.4 E\right)$. No condition difference was found for the think versus no-think contrast in the right $\operatorname{dlPFC}\left(t_{(50)}=1.99, p=0.052\right)$ and no 
A

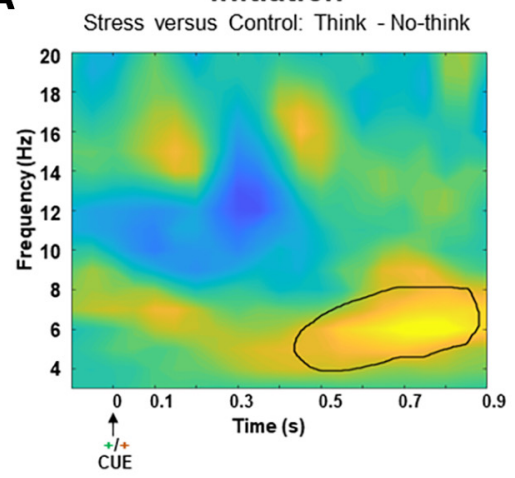

B

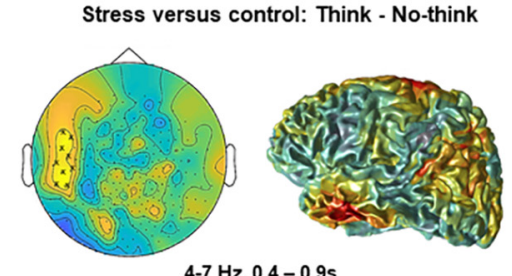

4-7 $\mathrm{Hz}, 0.4-0.9 \mathrm{~s}$

Inferior Temporal Lobe

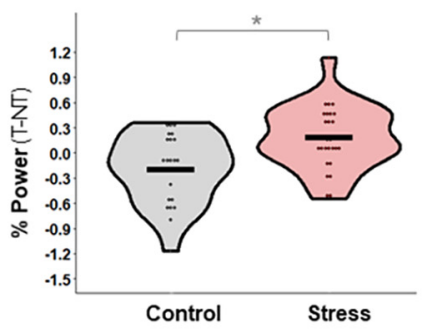

C

Intentional Control

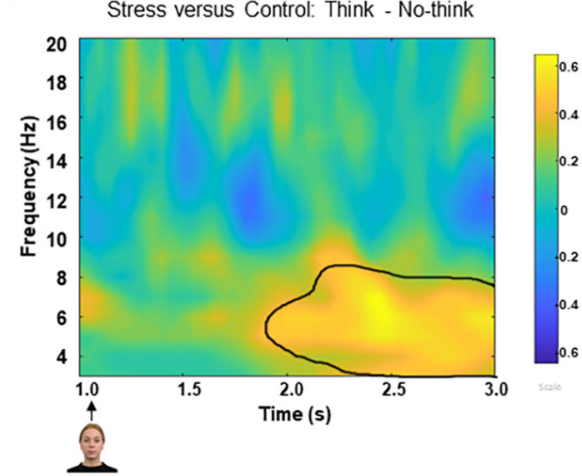

D

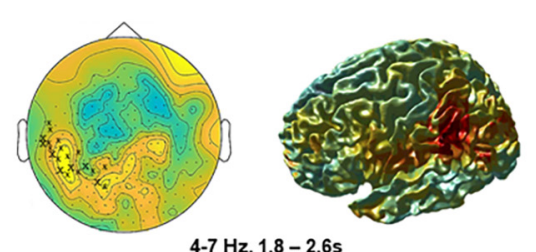

Inferior Parietal Cortex

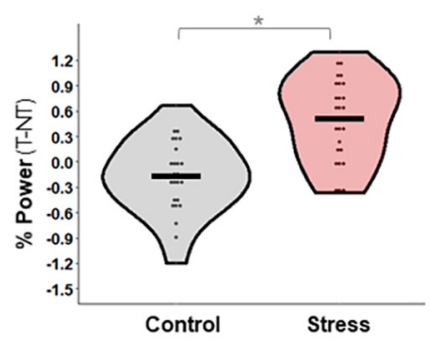

Intentional Control

Stress versus control: Think - No-think
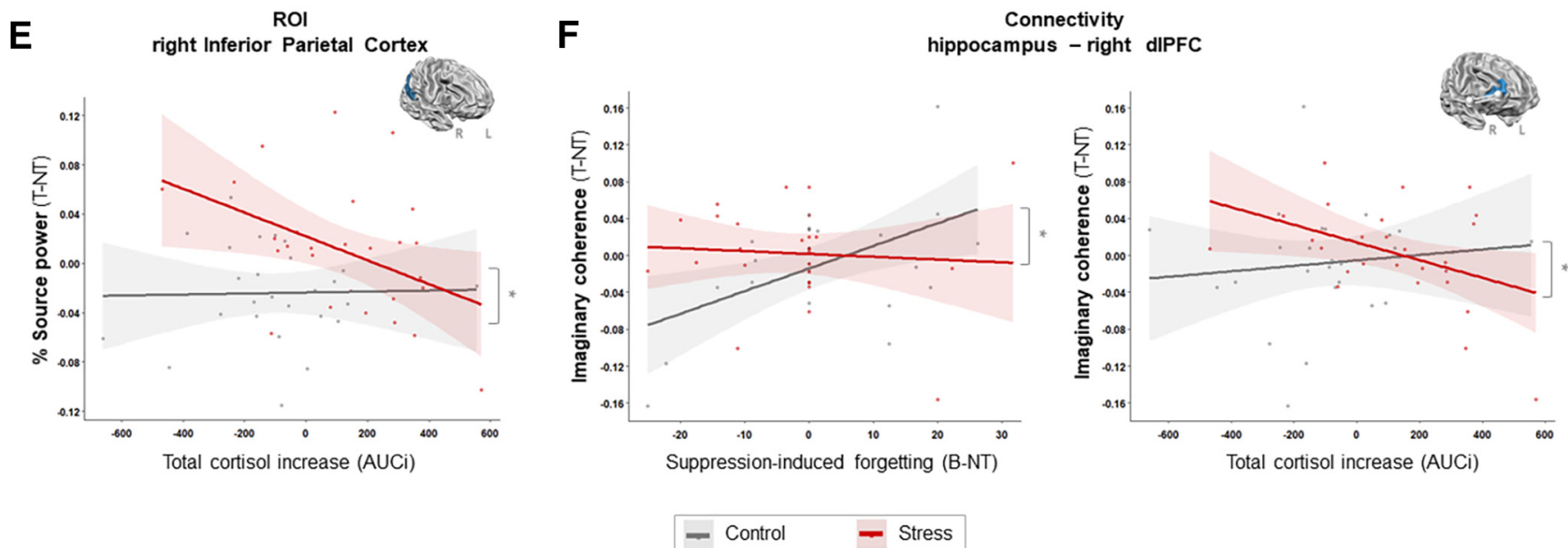

Figure 4. Differences in theta (4-7 Hz) activity for think and no-think face-word pairs between conditions. $A$, Time-frequency representation of sensor-level activity in the inferior temporal lobe for the initiation phase. Black contour lines indicate the result of the cluster-based permutation tests for think minus no-think for the condition contrast. $\boldsymbol{B}$, Topographical distribution of theta activity for think minus no-think using sensor topography and source localization. Stress modulated theta activity in the left inferior temporal lobe during the initiation phase (0.4-0.9 s). Significant cluster surviving permutation-based correction indicated with crosses. $C$, Time-frequency representation of sensor-level activity in the left inferior parietal cortex for the intentional control phase. Black contour lines indicate the result of the cluster-based permutation tests for think minus no-think for the condition contrast. $\boldsymbol{D}$, Topographical distribution of theta activity for think minus no-think using sensor topography and source localization. Stress modulated theta activity for think versus no-think items in the left lateral parietal cortex during the intentional control phase (1.8-2.4 s). E, Source power differences between conditions in the right inferior parietal cortex ROl for think versus no-think face-word pairs during the intentional control phase are differently associated with the cortisol increase (AUCi). $\boldsymbol{F}$, ROI-based hippocampal, right dIPFC connectivity was differently associated with suppression-induced forgetting and total cortisol increase in the control and stress condition. Violin plots display the distribution of the data, group means (indicated by the bar), and individual data points; $95 \% \mathrm{Cl}$ interval is displayed for the correlation lines; $N T=$ no-think word pair, $T=$ think word pair; ${ }^{*} p \leq 0.05$. 
associations with cortisol levels or behavioral data were found (all ps $>0.117$ ).

\section{Stress-induced modulation of functional connectivity between hippocampus and dlpfc linked to active memory suppression}

In order to investigate whether stress modulated hippocampal connectivity during intentional memory control, we assessed in a final step long-range phase synchronization in the theta band. To this end, we computed imaginary coherence within the theta band between the hippocampus and the right lateral PFC and right lateral parietal cortex. This analysis showed no condition differences in functional connectivity for the think versus nothink contrast between the hippocampus and right dlPFC or IPL (all ps $>0.355$ ). However, conditions differed significantly in the association between right hippocampus-right dlPFC connectivity and suppression-induced forgetting (baseline-no-think; $\mathrm{Z}=1.862, p=0.031$ ) and the cortisol increase (AUCi; $\mathrm{Z}=1.900$, $p=0.029$ ). Higher hippocampus-right dlPFC connectivity for think versus no-think items was associated with stronger suppression-induced forgetting in the control condition $\left(r_{(26)}=\right.$ $0.440, p=0.023$ ), while this association was non-significant in the stress condition $\left(r_{(27)}=-0.071, p=0.726\right.$; Fig. $\left.4 E\right)$. Furthermore, a higher cortisol increase was associated with decreased hippocampus-right dlPFC connectivity for think versus no-think items in the stress condition $\left(r_{(27)}=-0.416, p=0.031\right)$, whereas this association was not significant in the control condition $\left(r_{(26)}=0.111, p=0.590\right.$; Fig. $\left.4 F\right)$.

\section{Discussion}

Accumulating evidence points to the fascinating possibility that we can, to some degree, actively control our memories by retrieving some experiences while trying to suppress others (Anderson and Green, 2001; Anderson and Hanslmayr, 2014; Benoit et al., 2016; Depue et al., 2016; Ryckman et al., 2018). Using the wellestablished think/no-think paradigm (Anderson and Green, 2001; Hanslmayr et al., 2009), we tested here whether acute stress may interfere with the capacity to intentionally control memory. We demonstrate that stress interfered in particular with actively suppressing memories. Moreover, the inability to intentionally control memories under stress was linked to altered theta activity in the right IPL and to changes of in functional connectivity between the hippocampus and right dlPFC.

The ability to intentionally control memory has been demonstrated in several studies using the think/no-think paradigm (Anderson and Green, 2001; Anderson et al., 2004; Benoit and Anderson, 2012; Paz-Alonso et al., 2013; Benoit et al., 2015). In line with these earlier studies, the control group demonstrated higher recall of think compared with no-think face-word pairs reflecting intentional memory control. Most importantly, however, acute stress before the attempt to intentionally strengthen or suppress memories (i.e., the think/no-think phase) completely abolished the superior memory for think- relative to no-think items. Interestingly, stress impaired mainly the active suppression of memories, in line with the finding that stress impairs suppression-induced forgetting of future fears (Ashton et al., 2020). When not accompanied by noradrenergic activation, (genomic) glucocorticoid actions have been suggested to interfere with inhibitory processing (Shields et al., 2015). This is in line with the current finding of impaired suppression under stress and supports the notion that inhibitory control is needed for no-think items (Anderson and Hanslmayr, 2014; Anderson et al., 2016).
Furthermore, the fact that our data showed an effect that was specific to suppression-induced forgetting speaks clearly against the view that our findings represent another example for the well-known stress-induced retrieval changes (de Quervain et al., 1998, 2000, 2007; Gagnon and Wagner, 2016; Shields et al., 2017). If stress had affected retrieval per se, memory for retrieval (baseline vs think items) should have been affected as well, which was, however, not the case.

During the intentional control phase, acute stress modulated theta activity in the right inferior parietal cortex which was directly related to the cortisol response. In the stress condition, a more pronounced cortisol increase was associated with decreased right IPL theta activity for think versus no-think items, suggesting that cortisol might indeed be a driving force. This is in line with previous research suggesting that (genomic) glucocorticoid actions, when not accompanied by noradrenergic activation, reduce activity in a network including temporoparietal regions (Hermans et al., 2014). Corroborating the suggestion that the parietal cortex supports memory retrieval (Shimamura, 2011), we found a positive association between IPL theta activity and the retrieval index (baseline-think) in the control condition while this association was absent in the stress condition. The lateral parietal cortex has been found to be a key intermediary in the modulation of the medial temporal lobe by lateral PFC during memory suppression (Paz-Alonso et al., 2013).

Acute stress modulated the association between hippocampus-right dIPFC connectivity and suppression-induced forgetting and total cortisol increase. In control participants, stronger connectivity between the hippocampus and right dlPFC was associated with better forgetting, while this association was lacking in stressed participants. This stress-induced change in the association between hippocampal-dlPFC connectivity and memory suppression may well be driven by cortisol again as higher cortisol responses were associated with reduced theta phase synchronization between the hippocampus-right dlPFC. Theta phase synchronization has been considered an important indicator of top-down control and communication between the lateral PFC and medial temporal lobe, including the hippocampus (Vincent et al., 2006; Depue et al., 2013; Daume et al., 2017a,b). It should be noted however, that the present design does not allow an explicit dissociation of top-down and bottom-up processing.

During the initiation phase, when participants prepared to either reactivate or suppress a memory, stress modulated theta activity in the inferior temporal lobe. The think/no-think paradigm is based on visual memory in which the inferior temporal lobe plays a critical role via the medial temporal lobe (Axmacher et al., 2008). Moreover, the inferior temporal lobe has been suggested to work as an adaptive memory filter (Miller et al., 1991). The stress-induced modulation of theta activity found in the current study might imply a functionally impaired bottom-up memory filter under stress and thus the need for more control during the intentional control phase.

We focused here on the analysis of theta oscillations as these have been implicated in memory retrieval (Klimesch, 1999), maintenance (Nyhus and Curran, 2010), and intentional memory control (Ketz et al., 2014; Waldhauser et al., 2015), and a stress-induced reduction in theta oscillations has been demonstrated (Gärtner et al., 2014, 2015). Our results pave the way for future research as it is of high interest to show how theta power as a marker of deficient memory suppression under stress relates to power changes in other frequency ranges. Moreover, to obtain comparable signal-to-noise ratios between conditions, the think 
and no-think trial numbers where stratified. This resulted in a wide range of trial numbers with some participants having below 100 trials. Future studies should consider more strongly that for connectivity analyses and to detect activity from deep brain structures with MEG, a large number of trials results in more precise estimations (Quraan et al., 2011; Ruzich et al., 2019). Finally, it has to be noted that memory performance was overall very high in the final recall test, in line with previous studies using this think/no-think paradigm (Anderson and Green, 2001; Hanslmayr et al., 2009; Benoit and Anderson, 2012; Depue et al., 2013; Paz-Alonso et al., 2013; Ketz et al., 2014; Benoit et al., 2015; Waldhauser et al., 2015). This level of memory performance may have prevented stress effects on think (or baseline) items. However, the fact that intentional memory control resulted in a decrease for think relative to no-think items, despite the overall performance strength of memory, and that stress interfered with this memory control is a remarkable finding in itself.

In sum, our data show that acute stress impairs the intentional control of memory. This stress-induced deficit in intentional memory control was mainly linked to a modulation of theta power and corresponding source activity in the lateral parietal cortex, a region that mediates the executive control of the medial temporal lobe by lateral PFC during memory suppression (Paz-Alonso et al., 2013). Moreover, the stress-induced impairment in active memory suppression was linked to altered connectivity between the hippocampus and right dlPFC, which in turn was negatively correlated with stress-induced cortisol. These data point to conditions under which active memory control breaks down and may aid our understanding of stressrelated disorders, such as PTSD, in which patients are plagued by recurrent, vivid memories of aversive events and attempts to control these unwanted memories fail (Catarino et al., 2015; Waldhauser et al., 2018; Mary et al., 2020).

\section{References}

Anderson MC, Green C (2001) Suppressing unwanted memories by executive control. Nature 410:366-369.

Anderson MC, Levy BJ (2009) Suppressing unwanted memories. Curr Dir Psychol Sci 18:189-194.

Anderson MC, Huddleston E (2012) Towards a cognitive and neurobiological model of motivated forgetting. Nebr Symp Motiv 58:53-120.

Anderson MC, Hanslmayr S (2014) Neural mechanisms of motivated forgetting. Trends Cogn Sci 18:279-292.

Anderson MC, Ochsner KN, Kuhl B, Cooper J, Robertson E, Gabrieli SW, Glover GH, Gabrieli JD (2004) Neural systems underlying the suppression of unwanted memories. Science 303:232-235.

Anderson MC, Bunce JG, Barbas H (2016) Prefrontal-hippocampal pathways underlying inhibitory control over memory. Neurobiol Learn Mem 134 [Pt A]:145-161.

Ashton SM, Benoit R, Quaedflieg CWEM (2020) The impairing effect of acute stress on the intentional forgetting of future fears and its moderation by working memory capacity. Psychoneuroendocrinology 120:104790.

Axmacher N, Schmitz DP, Wagner T, Elger CE, Fell J (2008) Interactions between medial temporal lobe, prefrontal cortex, and inferior temporal regions during visual working memory: a combined intracranial EEG and functional magnetic resonance imaging study. J Neurosci 28:73047312.

Beck AT, Steer RA, Brown GK (1996) Beck depression inventory-II, pp 78204-72498. San Antonio: Psychological Corporation.

Benoit RG, Anderson MC (2012) Opposing mechanisms support the voluntary forgetting of unwanted memories. Neuron 76:450-460.

Benoit RG, Hulbert JC, Huddleston E, Anderson MC (2015) Adaptive topdown suppression of hippocampal activity and the purging of intrusive memories from consciousness. J Cogn Neurosci 27:96-111.
Benoit RG, Davies DJ, Anderson MC (2016) Reducing future fears by suppressing the brain mechanisms underlying episodic simulation. Proc Natl Acad Sci USA 113:E8492-E8501.

Catarino A, Küpper CS, Werner-Seidler A, Dalgleish T, Anderson MC (2015) Failing to forget: inhibitory-control deficits compromise memory suppression in posttraumatic stress disorder. Psychol Sci 26:604-616.

Daume J, Graetz S, Gruber T, Engel AK, Friese U (2017a) Cognitive control during audiovisual working memory engages frontotemporal theta-band interactions. Sci Rep 7:12585.

Daume J, Gruber T, Engel AK, Friese U (2017b) Phase-amplitude coupling and long-range phase synchronization reveal frontotemporal interactions during visual working memory. J Neurosci 37:313-322.

de Quervain DJ, Roozendaal B, McGaugh JL (1998) Stress and glucocorticoids impair retrieval of long-term spatial memory. Nature 394:787-790.

de Quervain DJ, Roozendaal B, Nitsch RM, McGaugh JL, Hock C (2000) Acute cortisone administration impairs retrieval of long-term declarative memory in humans. Nat Neurosci 3:313-314.

de Quervain DJ, Aerni A, Roozendaal B (2007) Preventive effect of beta-adrenoceptor blockade on glucocorticoid-induced memory retrieval deficits. Am J Psychiatry 164:967-969.

Debener S, Ullsperger M, Siegel M, Fiehler K, von Cramon DY, Engel AK (2005) Trial-by-trial coupling of concurrent electroencephalogram and functional magnetic resonance imaging identifies the dynamics of performance monitoring. J Neurosci 25:11730-11737.

Depue BE, Ketz N, Mollison MV, Nyhus E, Banich MT, Curran T (2013) ERPs and neural oscillations during volitional suppression of memory retrieval. J Cogn Neurosci 25:1624-1633.

Depue BE, Orr JM, Smolker HR, Naaz F, Banich MT (2016) The organization of right prefrontal networks reveals common mechanisms of inhibitory regulation across cognitive, emotional, and motor processes. Cereb Cortex 26:1634-1646.

Ebbinghaus H (1885) Ûber das Gedächtnis. Leipzig: Dunker.

Engen HG, Anderson MC (2018) Memory control: a fundamental mechanism of emotion regulation. Trends Cogn Sci 22:982-995.

Fan L, Li H, Zhuo J, Zhang Y, Wang J, Chen L, Yang Z, Chu C, Xie S, Laird AR, Fox PT, Eickhoff SB, Yu C, Jiang T (2016) The Human Brainnetome Atlas: a new brain atlas based on connectional architecture. Cereb Cortex 26:3508-3526.

Faul F, Erdfelder E, Lang AG, Buchner A (2007) G*Power 3: a flexible statistical power analysis program for the social, behavioral, and biomedical sciences. Behav Res Methods 39:175-191.

Gagnon SA, Wagner AD (2016) Acute stress and episodic memory retrieval: neurobiological mechanisms and behavioral consequences. Ann NY Acad Sci 1369:55-75.

Gärtner M, Rohde-Liebenau L, Grimm S, Bajbouj M (2014) Working memory-related frontal theta activity is decreased under acute stress. Psychoneuroendocrinology 43:105-113.

Gärtner M, Grimm S, Bajbouj M (2015) Frontal midline theta oscillations during mental arithmetic: effects of stress. Front Behav Neurosci 9:96.

Gómez-Ariza CJ, Iglesias-Parro S, Garcia-Lopez LJ, Díaz-Castela MM, Espinosa-Fernández L, Muela JA (2013) Selective intentional forgetting in adolescents with social anxiety disorder. Psychiatry Res 208:151-155.

Grandchamp R, Delorme A (2011) Single-trial normalization for eventrelated spectral decomposition reduces sensitivity to noisy trials. Front Psychol 2:236.

Gross J, Kujala J, Hamalainen M, Timmermann L, Schnitzler A, Salmelin R (2001) Dynamic imaging of coherent sources: studying neural interactions in the human brain. Proc Natl Acad Sci USA 98:694-699.

Hanslmayr S, Leipold P, Pastötter B, Bäuml KH (2009) Anticipatory signatures of voluntary memory suppression. J Neurosci 29:2742-2747.

Hanslmayr S, Volberg G, Wimber M, Oehler N, Staudigl T, Hartmann T, Raabe M, Greenlee MW, Bäuml KH (2012) Prefrontally driven downregulation of neural synchrony mediates goal-directed forgetting. J Neurosci 32:14742-14751.

Hermans EJ, Henckens MJ, Joëls M, Fernández G (2014) Dynamic adaptation of large-scale brain networks in response to acute stressors. Trends Neurosci 37:304-314.

Joëls M, Fernandez G, Roozendaal B (2011) Stress and emotional memory: a matter of timing. Trends Cogn Sci 15:280-288.

Joormann J, Hertel PT, LeMoult J, Gotlib IH (2009) Training forgetting of negative material in depression. Journal of Abnormal Psychology 118:3443. 
Ketz N, O’Reilly RC, Curran T (2014) Classification aided analysis of oscillatory signatures in controlled retrieval. Neuroimage 85 [Pt 2]:749-760.

Kirschbaum C, Pirke KM, Hellhammer DH (1993) The 'Trier Social Stress Test'-a tool for investigating psychobiological stress responses in a laboratory setting. Neuropsychobiology 28:76-81.

Klimesch W (1999) EEG alpha and theta oscillations reflect cognitive and memory performance: a review and analysis. Brain Res Brain Res Rev 29:169-195.

Ludowig E, Möller J, Bien CG, Münte TF, Elger CE, Rosburg T (2010) Active suppression in the mediotemporal lobe during directed forgetting. Neurobiol Learn Mem 93:352-361.

Maris E, Oostenveld R (2007) Nonparametric statistical testing of EEG- and MEG-data. Journal of Neuroscience Methods 164:177-190.

Martinez AM, Benavente R (1996) The AR face database. CVC Technical Report 24

Mary A, Dayan J, Leone G, Postel C, Fraisse F, Malle C, Vallée T, KleinPeschanski C, Viader F, de la Sayette V, Peschanski D, Eustache F, Gagnepain P (2020) Resilience after trauma: the role of memory suppression. Science 367:eaay8477.

Miller EK, Li L, Desimone R (1991) A neural mechanism for working and recognition memory in inferior temporal cortex. Science 254:1377-1379.

Nolte G (2003) The magnetic lead field theorem in the quasi-static approximation and its use for magnetoencephalography forward calculation in realistic volume conductors. Phys Med Biol 48:3637-3652.

Nolte G, Bai O, Wheaton L, Mari Z, Vorbach S, Hallett M (2004) Identifying true brain interaction from EEG data using the imaginary part of coherency. Clin Neurophysiol 115:2292-2307.

Norby S (2018) Forgetting and emotion regulation in mental health, anxiety and depression. Memory 26:342-363.

Nyhus E, Curran T (2010) Functional role of gamma and theta oscillations in episodic memory. Neurosci Biobehav Rev 34:1023-1035.

Oehrn CR, Fell J, Baumann C, Rosburg T, Ludowig E, Kessler H, Hanslmayr S, Axmacher N (2018) Direct electrophysiological evidence for prefrontal control of hippocampal processing during voluntary forgetting. Curr Biol 28:3016-3022.e4.

Oostenveld R, Fries P, Maris E, Schoffelen JM (2011) FieldTrip: open source software for advanced analysis of MEG, EEG, and invasive electrophysiological data. Comput Intell Neurosci 2011:156869.

Paz-Alonso PM, Bunge SA, Anderson MC, Ghetti S (2013) Strength of coupling within a mnemonic control network differentiates those who can and cannot suppress memory retrieval. J Neurosci 33:5017-5026.

Pruessner JC, Dedovic K, Khalili-Mahani N, Engert V, Pruessner M, Buss C, Renwick R, Dagher A, Meaney MJ, Lupien S (2008) Deactivation of the limbic system during acute psychosocial stress: evidence from positron emission tomography and functional magnetic resonance imaging studies. Biol Psychiatry 63:234-240.

Qin S, Hermans EJ, van Marle HJF, Luo J, Fernández G (2009) Acute psychological stress reduces working memory-related activity in the dorsolateral prefrontal cortex. Biol Psychiatry 66:25-32.

Quaedflieg CWEM, Schwabe L (2018) Memory dynamics under stress. Memory 26:364-376.

Quaedflieg CWEM, van de Ven V, Meyer T, Siep N, Merckelbach H, Smeets $\mathrm{T}$ (2015) Temporal dynamics of stress-induced alternations of intrinsic amygdala connectivity and neuroendocrine levels. PLoS One 10: e0124141.
Quraan MA, Moses SN, Hung Y, Mills T, Taylor MJ (2011) Detection and localization of hippocampal activity using beamformers with MEG: a detailed investigation using simulations and empirical data. Hum Brain Mapp 32:812-827.

Ruzich E, Crespo-García M, Dalal SS, Schneiderman JF (2019) Characterizing hippocampal dynamics with MEG: a systematic review and evidence-based guidelines. Hum Brain Mapp 40:1353-1375.

Ryckman NA, Addis DR, Latham AJ, Lambert AJ (2018) Forget about the future: effects of thought suppression on memory for imaginary emotional episodes. Cognition and Emotion 32:200-206.

Schacter DL (1999) The seven sins of memory. Insights from psychology and cognitive neuroscience. Am Psychol 54:182-203.

Schneider TR, Hipp JF, Domnick C, Carl C, Büchel C, Engel AK (2018) Modulation of neuronal oscillatory activity in the beta- and gamma-band is associated with current individual anxiety levels. Neuroimage 178:423434.

Schwabe L, Joëls M, Roozendaal B, Wolf OT, Oitzl MS (2012) Stress effects on memory: an update and integration. Neurosci Biobehav Rev 36:17401749 .

Schwibbe MH (1988) Der Semantische Atlas. Göttingen: Deutsches Primatenzentrum

Shields GS, Bonner JC, Moons WG (2015) Does cortisol influence core executive functions? A meta-analysis of acute cortisol administration effects on working memory, inhibition, and set-shifting. Psychoneuroendocrinology 58:91103.

Shields GS, Sazma MA, McCullough AM, Yonelinas AP (2017) The effects of acute stress on episodic memory: A meta-analysis and integrative review. Psychological Bulletin 143:636-675

Shimamura AP (2011) Episodic retrieval and the cortical binding of relational activity. Cogn Affect Behav Neurosci 11:277-291.

Smulders FTY, Ten Oever S, Donkers FCL, Quaedflieg CWEM, van de Ven V (2018) Single-trial log transformation is optimal in frequency analysis of resting EEG alpha. Eur J Neurosci 48:2585-2598.

Strahler J, Skoluda N, Kappert MB, Nater UM (2017) Simultaneous measurement of salivary cortisol and alpha-amylase: application and recommendations. Neurosci Biobehav Rev 83:657-677.

Tzourio-Mazoyer N, Landeau B, Papathanassiou D, Crivello F, Etard O, Delcroix N, Mazoyer B, Joliot M (2002) Automated anatomical labeling of activations in SPM using a macroscopic anatomical parcellation of the MNI MRI single-subject brain. Neuroimage 15:273-289.

Vincent JL, Snyder AZ, Fox MD, Shannon BJ, Andrews JR, Raichle ME, Buckner RL (2006) Coherent spontaneous activity identifies a hippocampal-parietal memory network. J Neurophysiol 96:3517-3531.

Vogel S, Kluen LM, Fernández G, Schwabe L (2018) Stress affects the neural ensemble for integrating new information and prior knowledge. Neuroimage 173:176-187.

Waldhauser GT, Bäuml KH, Hanslmayr S (2015) Brain oscillations mediate successful suppression of unwanted memories. Cereb Cortex 25:41804190.

Waldhauser GT, Dahl MJ, Ruf-Leuschner M, Müller-Bamouh V, Schauer M, Axmacher N, Elbert T, Hanslmayr S (2018) The neural dynamics of deficient memory control in heavily traumatized refugees. Sci Rep 8:13132.

Watson D, Clark LA, Tellegen A (1988) Development and validation of brief measures of positive and negative affect: the PANAS scales. J Pers Soc Psychol 54:1063-1070. 